\title{
Heroin-piracetam mixture: Suggested mechanisms of action and risks of misinterpretation for drug users
}

Medico-Legal Journa 0 (0) $1-4$

(C) The Author(s) 2017

Reprints and permissions: sagepub.co.uk/journalsPermissions.nav DOI: $10.1177 / 0025817217717846$ journals.sagepub.com/home/mlj SAGE

\author{
Simone Cappelletti ${ }^{1,2}$, Francesco Lombardo ${ }^{2}$, Pasquale Vitale ${ }^{2}$, \\ Giuseppe Vallone ${ }^{2}$ and Costantino Ciallella'
}

\begin{abstract}
Piracetam is a positive allosteric modulator of the $\alpha$-amino-3-hydroxy-5-methyl-4-isoxazolepropionic acid receptor that has been frequently used in the treatment of cognitive disorders. Press and internet reports indicate that the use of piracetam, as a heroin adulterant, has spread rapidly in some countries, especially in Asia and Europe. Its use, as adulterant, is believed to produce more profound desirable effects, while decreasing hangover. Recent surveys demonstrated that piracetam protects neurons from heroin-induced apoptosis. The protective role of this adulterating substance may be related to restoration of beta-endorphin levels and to its neuroprotective effects. The aim of this paper is to review the relevant literature and suggest the main hypothetical mechanisms that justify its use as a heroin adulterant, try to understand if its use could help people who want to come off heroin by reducing withdrawal symptoms and, finally, give useful information that permit us to understand why drug trafficking organisations started to use piracetam as heroin adulterant.
\end{abstract}

\section{Keywords}

Piracetam, heroin, withdrawal, body packer, body packing, forensic toxicology

\section{Introduction}

Piracetam (PIR), IUPAC name 2-(2-oxopyrrolidin-1yl)-acetamide, is a positive allosteric modulator of the $\alpha$-amino-3-hydroxy-5-methyl-4-isoxazolepropionic acid (AMPA) receptor, which improves behaviour, cognitive and memory functions and has been used as 'cognitive enhancer' clinically for decades. ${ }^{1}$ Press and internet reports indicate that the use of PIR, as a heroin adulterant, has spread rapidly in some countries, especially in Asia and Europe. ${ }^{2}$ Its use, as adulterant, is believed to produce more profound desirable effects, while decreasing hangover.

The use of PIR as a heroin adulterant has been reported in literature for a brief period, but there has been no attempt to explain how it works. ${ }^{3,4}$

Thus far, its addictive properties and potential effects to the central neural system are not known. Recent surveys demonstrated that PIR protects neurons from heroin-induced apoptosis. The protective role of this adulterating substance may be related to restoration of beta-endorphin levels and to its neuroprotective effects. This paper aims to review the relevant literature and suggest the main hypothetical mechanisms that justify its use as a heroin adulterant, try to understand if its use could help people who want to come off heroin by reducing withdrawal symptoms and, finally, offer some explanation on why drug trafficking organisations have started using PIR as heroin adulterant.

\section{Uses of PIR}

PIR is a member of the class of prescription drugs known as nootropics or 'smart drugs' and it is sold

\footnotetext{
'Legal Medicine Section - SAIMLAL Department, SAPIENZA University of Rome, Roma, Italy

${ }^{2}$ State Police Health Service Department, Ministry of Interior, Rome, Italy
}

\section{Corresponding author:}

Simone Cappelletti, Legal Medicine Section - SAIMLAL Department, SAPIENZA University of Rome, Viale Regina Elena, 336, 00161 Roma, Italy.

Email: simone.cappelletti@uniroma I.it 
under many brand names like Breinox ${ }^{\mathrm{TM}}$, Lucetam ${ }^{\mathrm{TM}}$, Nootropil $^{\mathrm{TM}}$, Oikamid ${ }^{\mathrm{TM}}$ or Piracetam ${ }^{\mathrm{TM}}$. ${ }^{5}$ It is a positive allosteric modulator of the AMPA receptor, which improves behaviour, cognitive and memory functions. Its use as 'cognitive enhancer' and potential treatment for neurological diseases has been known for decades. It has been widely used as a long-term treatment for clotting, coagulation and vasospastic disorders.

In the United States, PIR is not approved by the US Food and Drug Administration (FDA) for any medical use and may not be sold as a dietary supplement. In Europe, it is often used off-label for many conditions and as a dietary supplement. It is possible to buy PIR supplements, either in powder or in pill form online and in a pharmacy with a medical prescription. The average price per dose when purchasing PIR is $\$ 0.30$, making this one of the cheapest nootropics available for sale. Safety data on PIR have demonstrated that it has a low toxicity potential. Single doses of PIR yielded LD50 values at $26 \mathrm{~g} / \mathrm{kg}$ in mice but LD50 values were not reached in rats. In dogs, clinical signs after acute oral dosing were mild and lethality was not observed at the maximum tested dose of $10 \mathrm{~g} / \mathrm{kg}$; no tests were conducted on humans.

The FDA has a very strict definition for what qualifies as a dietary supplement. This policy is laid out in section 201 of the Food, Drug and Cosmetic Act, 21 U.S.C. $\S 321$. It states that a dietary supplement must be one of the following: a vitamin, mineral, amino acid, herbal extract, botanical compound or a concentrate, metabolite, constituent, extract or combination of a dietary ingredient. PIR is none of these things and therefore does not qualify as a supplement under FDA regulations. FDA considers, PIR to be pharmacologically active and so categorises it as a 'drug' but has not approved PIR as a nootropic compound because the FDA only gives regulatory approval to drugs intended to diagnose, treat, cure, or prevent diseases. PIR, at present, does not fall within the FDA's policy definition.

\section{PIR mechanisms of action}

The most accredited theories explaining how PIR works are:

- influence on level of dopamine;

- interaction with glutamatergic system;

- allosteric modulation of glutamatergic AMPA receptors;

- interaction with membrane phospholipids.

On the issue of dopamine action, animal studies have shown that PIR interferes with dopamine levels by increasing the stimulated dopamine release. $^{6}$
By contrast, lower doses of PIR demonstrated no significant influence on the dopaminergic brain activity. ${ }^{6,7}$

The PIR interaction with the glutamatergic system is not yet fully understood. As the glutamatergic system impairment was described in cognitive deficits, ${ }^{6,8}$ it is possible that glutamatergic effects of PIR contribute to its positive impact on cognition. Furthermore, PIR has an important role in the allosteric modulation of glutamatergic AMPA receptors, ${ }^{9}$ structures involved in positive reinforcement in addiction. ${ }^{10}$

The PIR interaction with membrane phospholipids is the most accredited mechanism of action on the activities of neurotransmitter systems. ${ }^{11}$ It is well known that neurotransmitter signals depend on binding to specific membrane protein structures, such as receptors and transporters. The PIR effect on the membrane fluidity could have a significant impact on binding sites for neurotransmitters and could, indirectly, affect their functioning. ${ }^{12}$

\section{Heroin-PIR known mechanisms of interaction}

The interaction between heroin and PIR may be explained in two different mechanisms.

The first, proposed by Wang et $a,,^{13}$ focuses on the hypoxic-ischemic brain damage and neuroinflammatory response that heroin causes to the central nervous system. It is well known that hypoxic-ischemic damage induces the release of chemokines, cytokines and other inflammatory mediators by the activation of leucocytes, ${ }^{14}$ endothelial and glial cells, ${ }^{15}$ especially microglia which is the predominant immune-competent cell type of the central nervous system. ${ }^{16}$ In response to these neurotoxic effects, PIR seems to increase activity of phospholipase A2, an intracellular messenger important in the production of arachidonic acid within the neuron. In turn, arachidonic acid is converted into prostaglandins, which can modulate neuronal excitability in a very general sort of way and thereby contribute to modulation of synaptic transmission. ${ }^{17}$ In studies examining neural injury in mice due to insufficient brain oxygenation, PIR has been shown to exert neuroprotective effects, indeed it increases synthesis of phospholipids, which help to protect neuronal and other brain membranes damages. The increase in synthesis of phospholipids requires high-energy compounds, for this reason, PIR increases energy reserves under reduced oxygen by maintaining normal adenosine triphosphate production. ${ }^{17}$

The second mechanism reported by Roth-Deri et al. ${ }^{18}$ is related to the opioid-receptor agonist action of heroin, that affect the levels of endogenous opioid peptides such as the beta-endorphin. Recent animal studies demonstrated that chronic heroin use resulted 


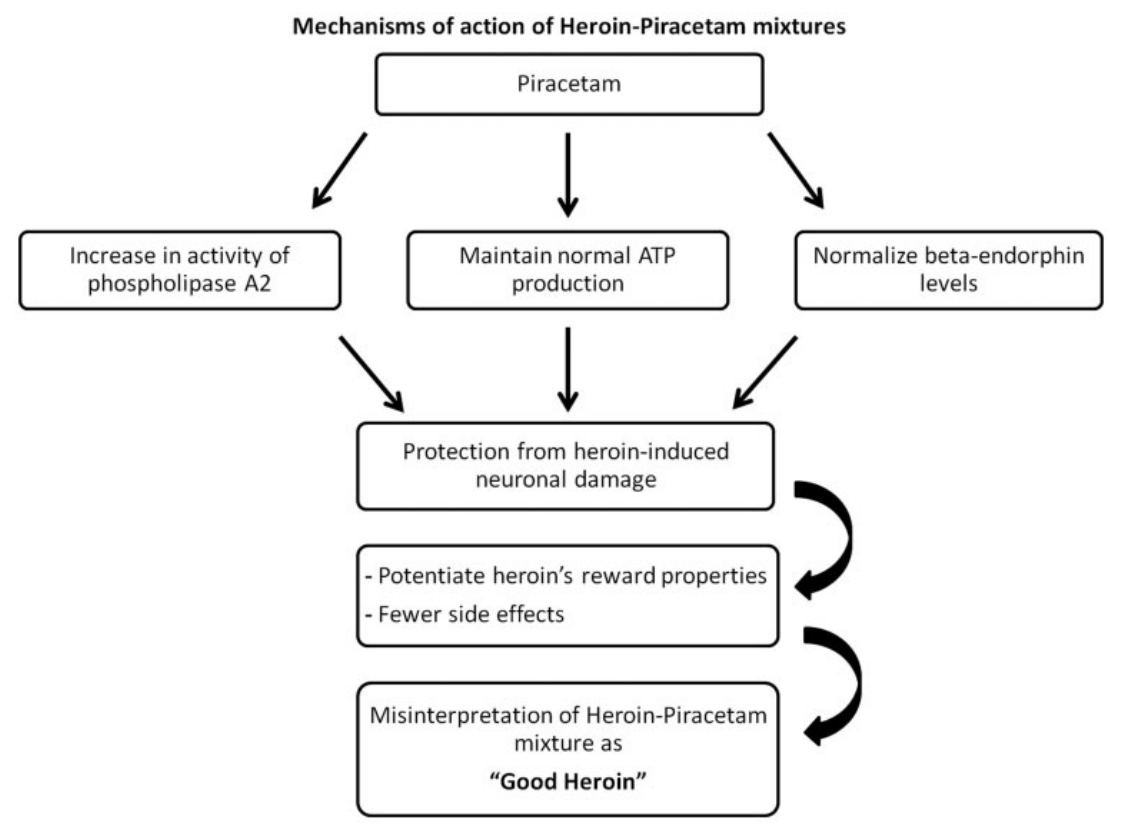

Figure I. Mechanisms of action of piracetam.

in a significant reduction of beta-endorphins, and in a heroin-induced neuronal apoptosis in the medial prefrontal cortex and nucleus accumbens within the corticolimbic system. The same authors showed how the treatment with PIR normalised the beta-endorphin levels and the neuronal morphologies in the abovementioned areas. ${ }^{19,20}$ These results imply that the neuroprotective role of PIR in heroin-induced apoptosis might be related to the restoration of beta-endorphin levels.

These mechanisms may affect the intensity and duration of heroin withdrawal symptoms. This was also confirmed in a paper by Chaudhry et al., ${ }^{21}$ in which the authors analysed aches and pains, fatigue and drowsiness among two heroin addicted groups treated with PIR and placebo, respectively. They concluded that in the group treated with PIR, the said symptoms disappeared more quickly than in the placebo group, confirming a possible role for PIR in heroin users.

The aforementioned mechanisms of action could affect drug users differently. For example, there is some evidence to suggest that PIR could help people who want to come off heroin and reduce withdrawal symptoms. However, there is no definitive data on this, but it is possible that it may be useful and more research is indicated.

Drug trafficking organisations appear to use this mixture to encourage drug use by its effect of reducing the malign consequences for the user which would explain why the supply of heroin adulterated with PIR has spread rapidly in recent years.

\section{Conclusion}

The use of PIR as an adulterant needs more study to enable us to have a clearer understanding of how it works. Preliminary hypotheses have been put forward and we have summarised the ways in which we believe PIR may operate to reduce the effects of heroin on the central nervous system (Figure 1).

The results of the study suggest that the combination of heroin-PIR makes drug addicts feel more comfortable and suffer fewer side effects. There is a danger that heroin addicts might misinterpret this type of drug mixture as 'good heroin'. In particular, these studies demonstrated that this mixture, even in low doses, improves heroin's reward properties and reduces its effects on the central nervous system.

That said, it seems to us there is real potential to use the combination to assist people to withdraw from heroin addiction more easily with fewer ill effects. The other side of the coin is that the option of withdrawal may seem less attractive if continued heroin use is less destructive to the addict.

Further, we need to understand why drug trafficking organisations are using PIR as a heroin adulterant, and these studies offer a useful starting point. But further research on this topic is needed.

\section{References}

1. Stockburger C, Kurz C, Koch KA, et al. Improvement of mitochondrial function and dynamics by the metabolic enhancer piracetam. Biochem Soc Trans 2013; 41: 1331-1334. 
2. Cappelletti S, Aromatario M, Bottoni E, et al. Drugrelated deaths with evidences of body packing: two case reports and medico-legal issues. Leg Med (Tokyo) 2016; 20: $23-26$.

3. de la Fuente L, Saavedra P, Barrio G, et al. Temporal and geographic variations in the characteristics of heroin seized in Spain and their relation with the route of administration. Spanish Group for the Study of the Purity of Seized Drugs. Drug Alcohol Depend 1996; 40: 185-194.

4. Chaudron-Thozet H, Girard J and David JJ. Analysis of heroin seized in France. Bull Narc 1992; 44: 29-33.

5. Cakic V. Smart drugs for cognitive enhancement: ethical and pragmatic considerations in the era of cosmetic neurology. J Med Ethics 2009; 35: 611-615.

6. Slais K, Machalova A, Landa L, et al. Could piracetam potentiate behavioural effects of psychostimulants? Med Hypotheses 2012; 79: 216-218.

7. Bhattacharya SK, Upadhyay SN, Jaiswal AK, et al. Effect of piracetam, a nootropic agent, on rat brain monoamines and prostaglandins. Indian $J$ Exp Biol 1989; 27: 261-264.

8. Ernst T, Jiang CS, Nakama H, et al. Lower brain glutamate is associated with cognitive deficits in HIV patients: a new mechanism for HIV associated neurocognitive disorder. J Magn Reson Imaging 2010; 32: 1045-1053.

9. Ahmed $\mathrm{AH}$ and Oswald RE. Piracetam defines a new binding site for allosteric modulators of alpha-amino-3hydroxy-5-methyl-4-isoxazole-propionic acid (AMPA) receptors. J Med Chem 2010; 53: 2197-2203.

10. Bowers MS, Chen BT and Bonci A. AMPA receptor synaptic plasticity induced by psychostimulants: the past, present, and therapeutic future. Neuron 2010; 67: 11-24.

11. Muller WE, Koch S, Scheuer K, et al. Effects of piracetam on membrane fluidity in the aged mouse, rat, and human brain. Biochem Pharmacol 1997; 53: 135-140.
12. Winblad B. Piracetam: a review of pharmacological properties and clinical uses. CNS Drug Rev 2005; 11: 169-182.

13. Wang X, Loram LC, Ramos K, et al. Morphine activates neuroinflammation in a manner parallel to endotoxin. Proc Natl Acad Sci USA 2012; 109: 6325-6330.

14. Loram LC, Grace PM, Strand KA, et al. Prior exposure to repeated morphine potentiates mechanical allodynia induced by peripheral inflammation and neuropathy. Brain Behav Immun 2012; 26: 1256-1264.

15. Allan SM and Rothwell NJ. Inflammation in central nervous system injury. Philos Trans $R$ Soc Lond B: Biol Sci 2003; 358: 1669-1677.

16. Schwarz JM, Hutchinson MR and Bilbo SD. Early-life experience decreases drug-induced reinstatement of morphine CPP in adulthood via microglial-specific epigenic programming of anti-inflammatory IL-10 expression. J Neurosci 2011; 31: 17835-17847.

17. McDaniel MA, Maier SF and Einstein GO. "Brain-specific" nutrients: a memory cure? Nutrition 2003; 19: 957-975.

18. Roth-Deri I, Green-Sadan T and Yadid G. Beta-endorphin and drug-induced reward and reinforcement. Prog Neurobiol 2008; 86: 1-21.

19. Maslov LN, Oeltgen PR, Lishmanov YB, et al. Activation of peripheral delta opioid receptors increases cardiac tolerance to arrhythmogenic effect of ischemia/ reperfusion. Acad Emerg Med 2014; 21: 31-39.

20. Tanaka K, Kersten JR and Riess ML. Opioid-induced cardioprotection. Curr Pharm Des 2014; 20: 5696-5705.

21. Chaudhry HR, De Mahieu C, Javaid MA, et al. The effect of piracetam in the detoxification of heroin dependents. Curr Ther Res Clin Exp 1990; 48: 313-319. 